\title{
"investigación en enseñanza de la carrera educación básica" Guayaquil, ecuador, 18 y 19 de agosto del 2017
}

\section{Proyecto educativo enfocado al arte y creatividad dentro de mi comunidad "recorriendo mi mercado"}

DOI: $10.46932 / \mathrm{sfjdv2n4-086}$

Received in: March 1st, 2021

Accepted in: May 30th, 2021

\author{
Sandra Patricia Chantong Drouet \\ Máster Universitario En Formación Internacional Especializada Del Profesorado. Universidad \\ Complutense De Madrid. \\ Institución actual: escuela de educación básica fiscal veinticuatro de mayo \\ Domicilio completo:av.9 de octubre 1312 y av. Quito, ciudad: guayaquil -ecuador \\ Correo electrónico: schantong2011@ hotmail.com \\ Maira Janeth Monar Pazmiño \\ Máster Universitario En Formación Internacional Especializada Del Profesorado. Universidad \\ Complutense De Madrid. \\ Institución actual: escuela de educación básica fiscal "rafaela vallejo barahona" \\ Domicilio completo: josé maría egas y rodolfo baquerizo nazur alborada 5ta etapa mz. Ih villa 13 \\ Correo electrónico: maira_monar1@hotmail.es \\ Gloria Verónica Parreño Arellano \\ Máster Universitario En Formación Internacional Especializada Del Profesorado. Universidad \\ Complutense De Madrid. \\ Institución actual: escuela de educación básica emilio clemente huerta \\ Domicilio completo: $\mathrm{km} 12$ 1/2 vía a daule urbanización "el caracol, manzana 618 villa 5" \\ Correo electrónico: gparreno61@ @otmail.com
}

\begin{abstract}
RESUMEN
Conocedoras de la relevancia que tiene la educación infantil en la vida de los seres humanos, etapa en la que se desarrolla su inteligencia, personalidad, comportamiento social y otras potencialidades, consideramos que es importante hacer cambios en nuestra labor educativa y aplicar métodos activos de enseñanza- aprendizaje, promoviendo la participación de los estudiantes en proyectos de aula, trabajo colaborativo y a partir de la experiencia crear su propio conocimiento con la guía de los docentes. Nuestra propuesta es la visita al mercado del barrio con la finalidad de que conozcan su comunidad y de que se sientan orgullosos de su cultura y costumbres; destacar la diferencia entre la comida nutritiva y alimentos chatarra; reconocer la labor que realizan los vendedores en el mercado; resaltar valores como la honestidad y el respeto; conocer las reglas del aseo para la conservación de los alimentos y cuidado del medio ambiente. Aplicar esta estrategia activa permite utilizar de manera apropiada recursos de nuestro entorno, crear el sentido de pertenencia, mejorar el desempeño escolar, la participación creativa de los estudiantes en cada uno de los ambientes de aprendizajes y aumentar su capacidad lingüística.
\end{abstract}

Palabras claves: Expresión artística, contexto social y cultural. 


\begin{abstract}
Knowing the relevance of the education of children in the life of human beings, a stage in which their intelligence, personality, social behavior and other potential develops, we consider that it is important to make changes in our educational work and to apply active teaching methods - learning, promoting the participation of students in classroom projects, collaborative work and based on the experience, to create their own knowledge with the guidance of teachers. Our proposal is the visit to the market of the neighborhood in order that they know their community and that they are proud of their culture and customs; to recognize the difference between nutritious food and junk foods; Recognize the work of marketers in the market; to exalt values such as honesty and respect; to know the rules of cleanliness for food preservation and care of the environment. Applying this active strategy allows us to appropriately use resources from our environment, create a sense of belonging, improve school performance in students, creative participation of students in every learning environment and increase their language ability.
\end{abstract}

Key words: Artistic expression, social and cultural context.

\title{
1 INTRODUCCIÓN
}

Este proyecto ha sido pensado para resaltar la importancia de valorar nuestra cultura en los grupos de niños de todas las edades a través del contacto directo con el entorno social - cultural, fomentar la participación en grupos de trabajo, compartir materiales y respetar opiniones de los compañeros.

La presentación de este proyecto responde a la necesidad de aplicar nuevas metodologías para que los estudiantes desarrollen la sensibilidad por la apreciación del arte mediante el mundo de la expresión escénica, primero en forma individual y luego grupal, que sean capaces de reproducir momentos relacionados con la visita al mercado.

Este trabajo aporta muchas ventajas al estudiante, al realizar un trabajo original adquiere aprendizajes que le servirá para aplicarlo en las diferentes tareas que representen la vivencia experimentada en el mercado.

Hemos utilizado el método lúdico, vivencial, globalizador y creativo con los cuales se ha desarrollado destrezas, habilidades, imaginación, creatividad en la expresión oral y escrita. La expresión plástica, el dramatizado y la práctica de valores ponen en evidencia lo aprendido.

La participación e involucramiento de los actores de la comunidad educativa son un referente importante para el desarrollo exitoso del proyecto.

Finalmente obtener los resultados de la experiencia responde a los objetivos planteados en nuestra propuesta. 


\section{DESARROLLO}

\subsection{FUNDAMENTACIÓN TEÓRICA DE LA PROPUESTA.}

La necesidad de reforzar en los estudiantes la importancia de valorar nuestra cultura forma parte de nuestro programa y es el mejor momento para realizar este novedoso proyecto el mismo que pueden realizar niños de todas las edades y con mayor curiosidad los más pequeños desarrollando su imaginación, su creatividad en las diferentes actividades que se realicen, es necesario fomentar la participación en grupos de trabajo para compartir materiales y a la vez respetar opiniones de los compañeros.

\subsection{FUNDAMENTACIÓN ARTÍSTICA.}

El arte a través de la Expresión Corporal, Teatro, Música y Expresión Plástica, constituyen el lenguaje simbólico, cada una de ellas permite que los estudiantes se acerquen paulatinamente al conocimiento y al uso de los códigos del lenguaje que permitirán la representación gráfica musical, dramática propiciando el desarrollo de aprendizajes significativos, ayuda a desarrollar la creatividad, imaginación, destrezas en las actividades diarias.

\subsection{FUNDAMENTACIÓN PEDAGÓGICA.}

Las acciones educativas que promueve el Proyecto están cimentadas en un eje de flexibilidad cuyos criterios permiten el desarrollo de diversos procesos; propician la participación de los actores involucrados, y a partir de la experiencia generada por éstos se reconocen las alternativas posibles para recrear y reorientar dichos procesos. Sin embargo, para el Proyecto Educativo los procesos históricos adquieren relevancia en la medida que surge de lo cotidiano, ya que la realidad es una construcción social y cultural que parte del presente de los individuos y las colectividades. En consecuencia, el Proyecto recupera lo más valioso de la tradición educativa, la cual se ve resignificada por los retos que exige a la educación el contexto social, cultural, ecológico, político y económico del mundo actual.

\section{METODOLOGÍA}

Hemos utilizado el método lúdico, vivencial, globalizador y creativo, con la participación activa de autoridades, maestra, estudiantes y padres de familia/ representantes.

\section{Técnicas activas de enseñanza - aprendizaje:}

- Lluvia de ideas

- Aprendizaje por investigación

- Descripción de láminas 
- Observación directa visita al mercado

- Dramatizaciones

- Juego-trabajo

- Mapas conceptuales

- Técnicas grafo-plásticas

- Exposición.

\section{Estrategias}

- Motivación para que los niños busquen información acerca de los mercados (Con ayuda de los padres de familia).

- En la visita al Mercado se aplican varias estrategias:

- Respetar las señales de tránsito y el cuidado del medio ambiente al trasladarse al mercado.

- Saludar a las personas que están en el mercado,

- Observar los diferentes productos que se expenden, sus colores, formas, tamaños, texturas, sabores.

- Observar la relación comercial entre los expendedores y los clientes.

- Uso de la moneda como medio de comercialización.

- Respeto por los espacios públicos y la señalética.

\section{Puesta en común en el salón de clases.}

- Conversación en forma detallada sobre lo observado en el mercado.

- Dramatización de escenas sobre compra y venta de alimentos.

- Reproducción de lo observado con técnicas grafo-plásticas plasmando la experiencia en armados, modelados, pintura.

- Exposición de los trabajos realizados por los niños.

\section{Involucrados}

- Autoridades y maestra

- Estudiantes del subnivel Preparatoria

- Padres de Familia

\section{Espacios}

- Visita al Mercado "San Joaquín"

- Salón de clases

- Patio del Plantel 


\section{Resultado de la experiencia}

- Desarrollo de nuevas técnicas de expresión lingüística.

- Seguridad para expresarse en público.

- Socialización (Oportunidad para trabajar en grupos.)

- Refuerzo de valores: respeto, compartir, responsabilidad, honestidad etc.

- Desarrollo de imaginación, creatividad.

- Descubrimiento de estudiantes con habilidades innatas al declamar un poema, un posible actor etc.

- Fortalecimiento de las capacidades cognitivas.

- Desarrollo de las inteligencias múltiples.

- Desarrollo de psicomotricidad fina y gruesa.

- Integración de la familia en las actividades de este proyecto.

- Difusión de la importancia de nuestra cultura.

- Participación activa de los estudiantes en todas las actividades.

- Reconocimiento de los alimentos nutritivos necesarios para nuestra alimentación. (Frutas, legumbres, verduras, víveres, carnes, lácteos, etc.)

- Valora la importancia del trabajo que realizan las personas que trabajan en el mercado.

- Fomento de hábitos de limpieza, orden.

\section{CONCLUSIONES}

\section{A partir de esta propuesta podemos concluir que:}

- Este proyecto representa el cambio de los paradigmas tradicionales por los métodos activosparticipativos dentro del marco de una educación innovadora que busca favorecer al estudiante brindándole herramientas de carácter significativo para desenvolverse en la vida diaria.

- Los principales actores son los educadores y estudiantes que participan de un cambio en este proceso de inter-aprendizaje, que es considerado de gran importancia para desarrollar las destrezas, aumentar el interés y la dedicación en cada situación de aprendizaje.

- En pleno siglo XXI con el avance de la tecnología, el aprendizaje debe estar basado en las experiencias y en la construcción del conocimiento apoyado en entornos culturales y sociales.

- La estrategia metodológica desarrolla destrezas, habilidades, imaginación, creatividad en la expresión oral y escrita a través del área de expresión plástica, dramatización y refuerza valores que le permiten vivir mejor en comunidad. 
- Aplicar las metodologías activas con los niños ha provocado aprendizajes constructivos para su vida y para su entorno. La educación tradicional queda en el pasado y por el contrario ahora es mucho más entretenida, divertida y enriquecedora.

A partir de esta experiencia es recomendable:

La toma de conciencia de nosotros los Docentes y el cambio de actitud para aplicar una educación innovadora, científica y tecnológica, la cual será el punto de partida para obtener los aprendizajes significativos necesarios para la vida y para el desarrollo de una sociedad.

Promover en esta sociedad del Siglo XXI la formación de sujetos críticos, reflexivos, participativos, empoderados de su conocimiento y conscientes del uso que le puedan dar a ese conocimiento para contribuir con los avances científicos y tecnológicos, con respeto del cuidado del medio ambiente y del entorno, con bases morales, éticas, con principios de igualdad, equidad, justicia y libertad. 


\section{BIBLIOGRAFÍA DEL PROYECTO}

- Guía para elaborar proyectos de pre-escolar

- $\quad$ http://unesdoc.unesco.org/images/0007/000720/072067SB.pdf

- $\quad$ bdigital.uncu.edu.ar/objetos_digitales/1098/fproyectosc.pdf

- hekademos.com/.../Proyecto_de_habitos_de_vida_saludable_en_Educaci..

- www.monografias.com > Educación

- $\quad$ www.educacioninicial.com/

- $\quad$ www.monografias.com/trabajos10/gotsky/gotsky.shtml

- $\quad$ LA PROPUESTA DE VYGOTSKY: LA PSICOLOGÍA SOCIOHISTÓRICA1

FOTOGRAFÍAS DE LA EXPERIENCIA

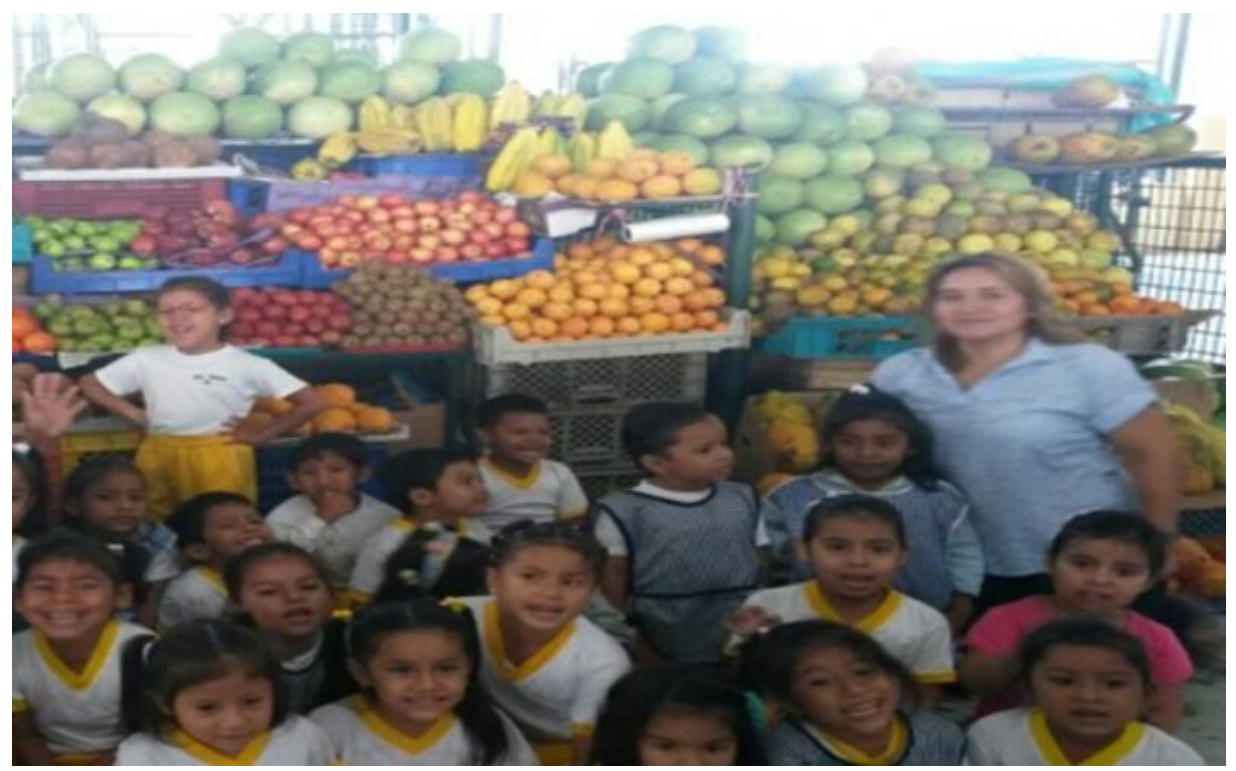

Visita al Mercado "San Joaquín” 
Observando los productos en el mercado.

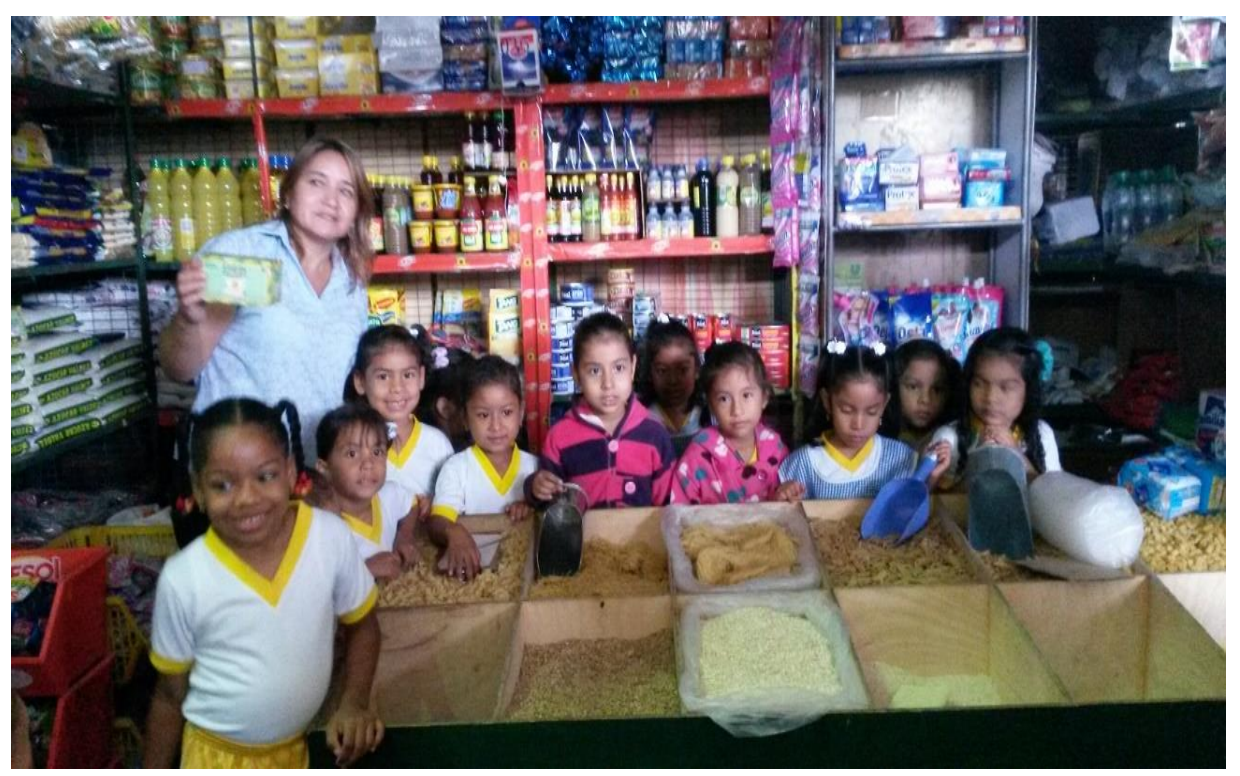

Comparando los productos de venta en el mercado.

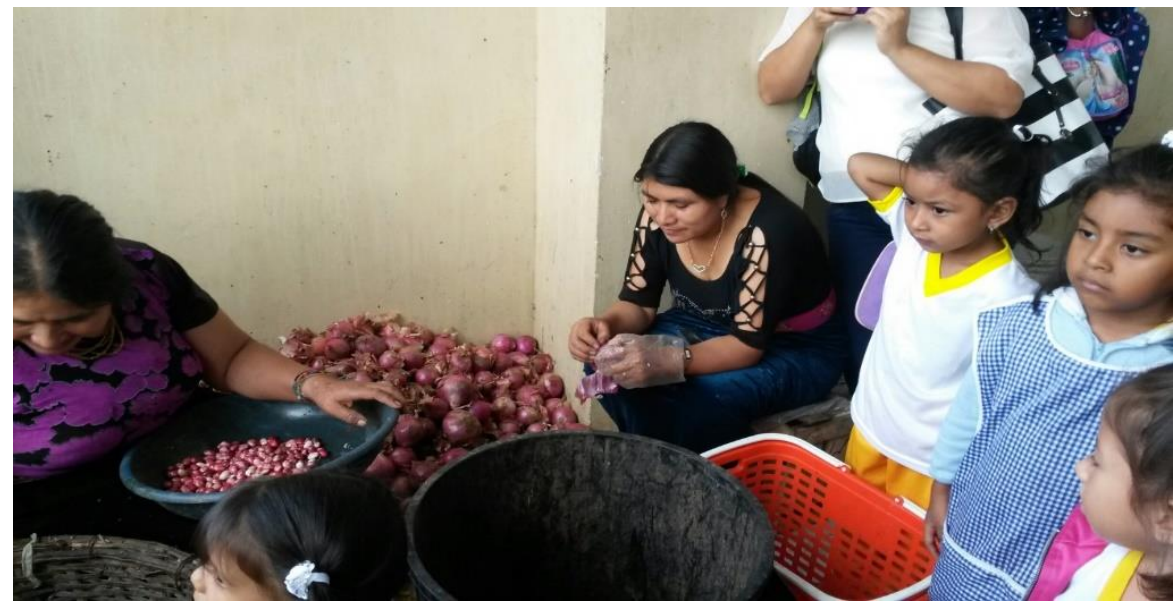

Observando el proceso de limpieza y selección de los productos por los expendedores del mercado. 

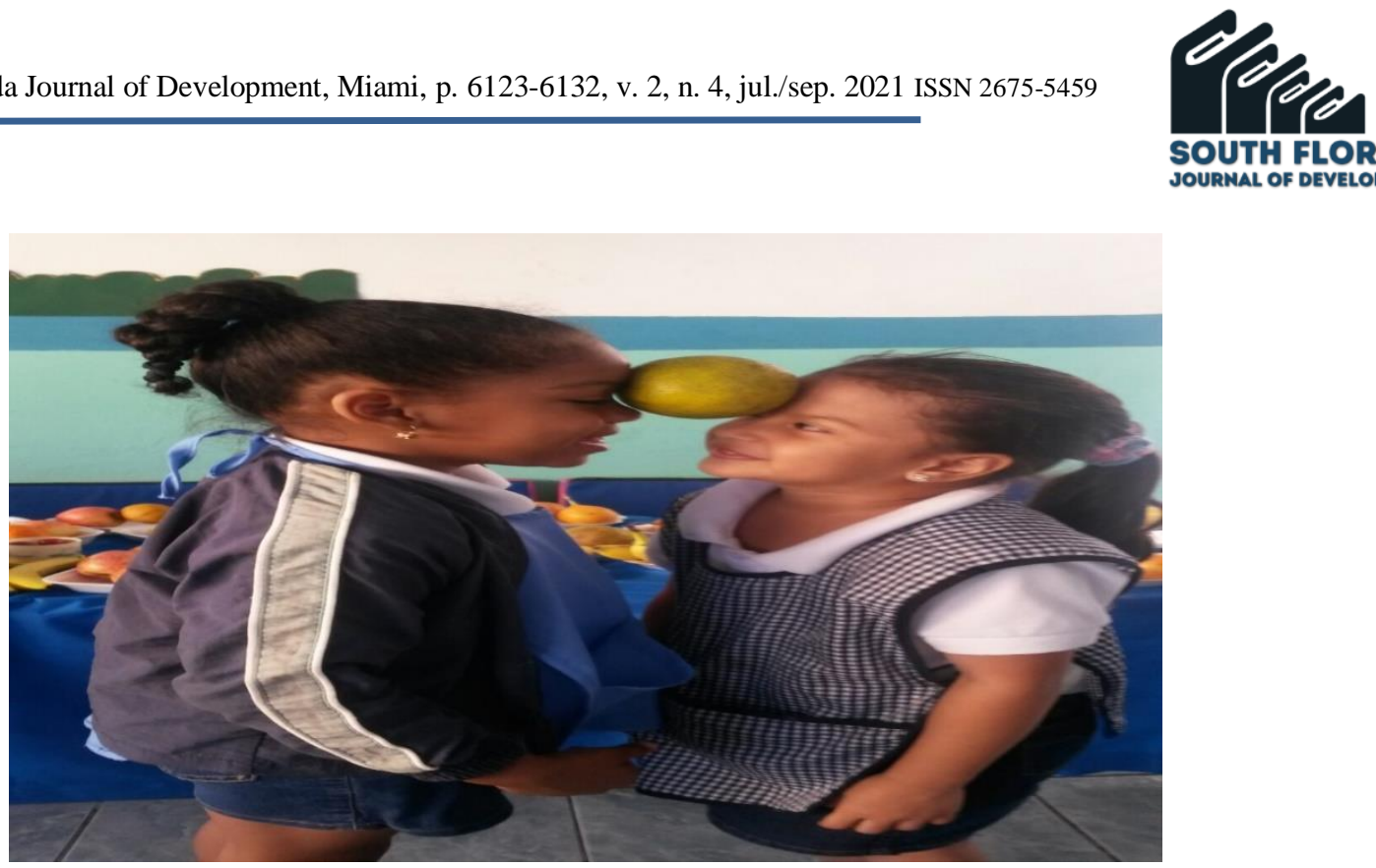

Creando con su cuerpo y las frutas diferentes mensajes

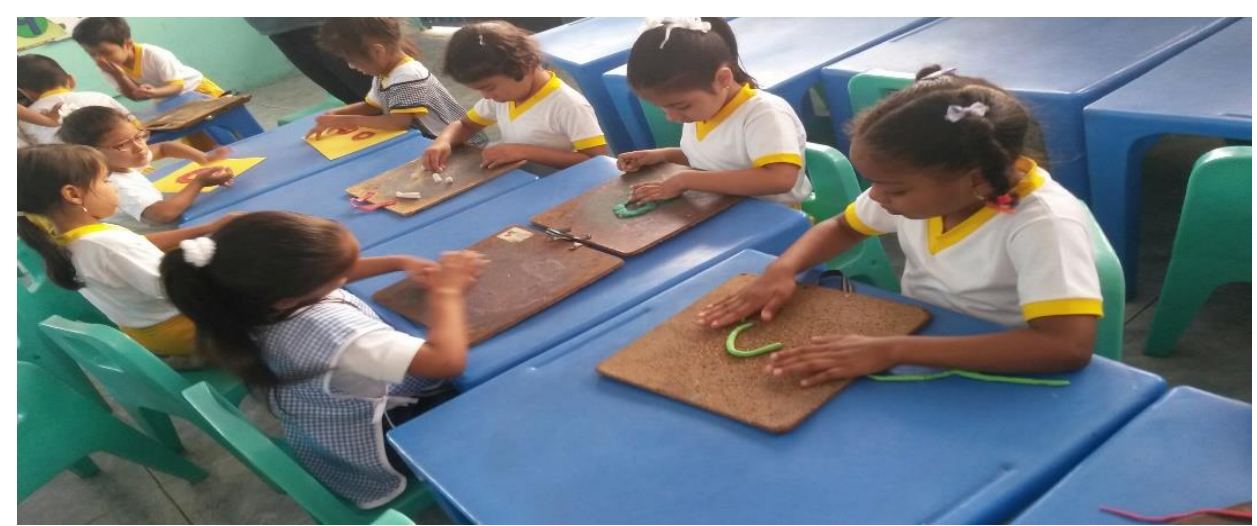

Modelando con plastilina los productos que observaron en el mercado.

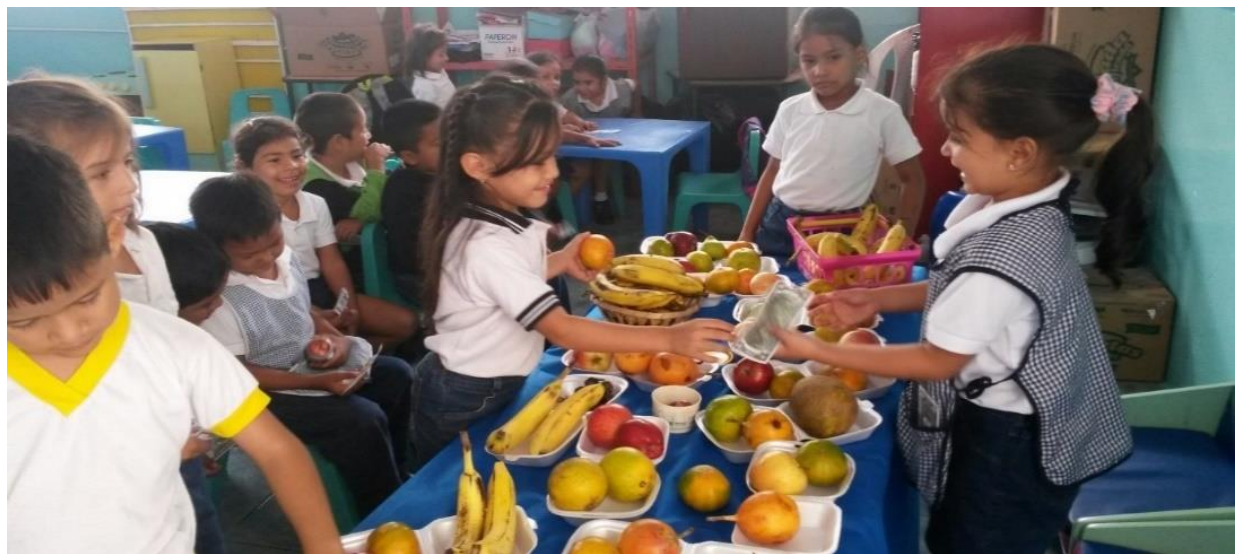

Representación dramática sobre la compra - venta de productos en el salón de clases. 


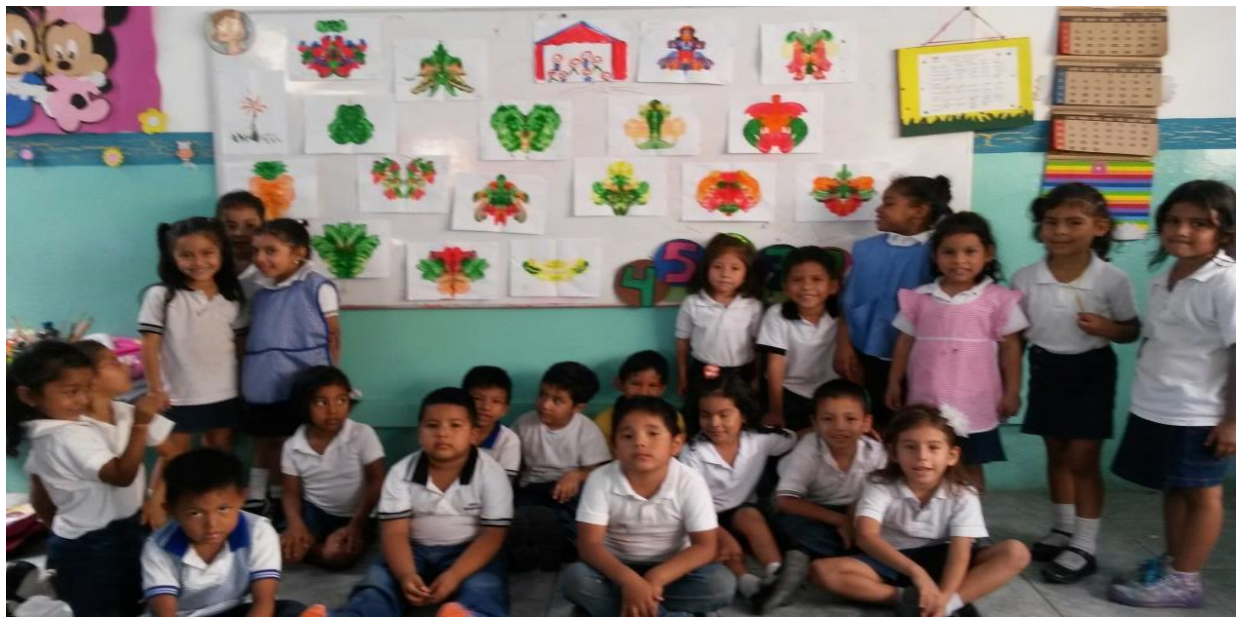

Exposición de los trabajos con técnicas grafo- plásticas en el salón de clases. 